\title{
THE MASS-LUMINOSITY RELATION FROM BINARIES
}

\author{
DAVID W. LATHAM \\ Harvard-Smithsonian Center for Astrophysics \\ 60 Garden Street, Cambridge, Massachusetts, 02138, USA
}

What is known about the masses of main-sequence stars from the analysis of binary orbits? Doublelined eclipsing binaries are the main source of very precise stellar masses and radii (e.g. Andersen 1997), contributing more than 100 determinations with better than $2 \%$ precision over the range 0.6 to $20 M_{\odot}$. For lower-mass stars we are forced to turn to nearby systems with astrometric orbits (e.g. Henry et al. 1993). Not only is the number of good mass determinations from such systems smaller, but also the precision is generally poorer. We are approaching an era when interferometers should have a major impact by supplying good astrometric orbits for dozens of double-lined systems. Already we are beginning to see the sorts of results to expect from this (e.g. Torres et al. 1997).

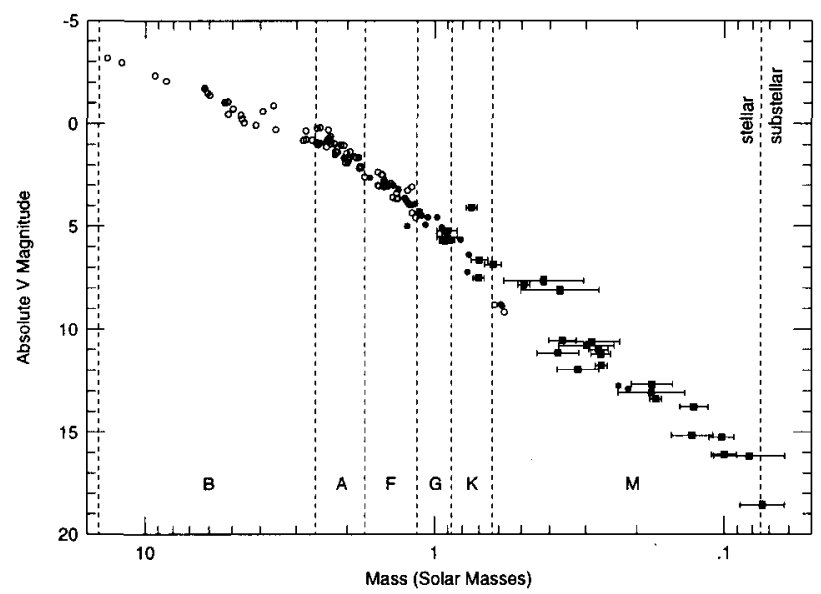

Figure 1. Mass vs. absolute $V$ magnitude for eclipsing binaries (circles) and nearby astrometric binaries (squares)

Figure 1 is an updated version of a diagram presented by Henry et al. (1993, their Figure 2). It shows the general run of mass determinations from about $10 M_{\odot}$ down to the substellar limit near $0.075 M_{\odot}$. Ninety of the points in Figure 1 are for eclipsing binary masses from Andersen's review (1991) and are plotted as open circles. The results for eclipsing binaries published since 1991 are plotted as 30 filled circles, adopting the same limit of $2 \%$ for the mass precision. In most cases the uncertainties are similar to the size of the symbols. Especially noteworthy is the pair of new points for CM Draconis (Metcalfe et al. 1996) with masses near $0.25 M_{\odot}$. Together with the points for YY Geminorum near $0.6 M_{\odot}$, these are the only $\mathrm{M}$ dwarfs that have precise mass determinations. For the most part we are forced to rely on nearby stars with astrometric orbits, to fill in the $M$ dwarf region of the diagram. We have used filled squares in. Figure 1 for 29 such systems from Henry et al. (1993), updated using 14 new parallaxes from Hipparcos and 4 from the new Yale Parallax Catalog (1995). Gliese 508 is not included, because it is now known to be a triple, while Gliese $67 \mathrm{AB}, 570 \mathrm{BC}$, and $623 \mathrm{AB}$ are not included because there are not yet any direct measurements of the $V$ magnitude difference for these systems. 
For eclipsing binaries it is normally possible to derive good values for the luminosities, if suitable photometry is available for deriving a reliable effective temperature, because the stellar radius comes directly out of the orbital solution. However, for the cool stars the bolometric corrections are rarely well determined, and the usual practice is to quote absolute magnitudes rather than luminosities. I have chosen to use the absolute magnitude in the $V$ band for the vertical axis in Figure 1.

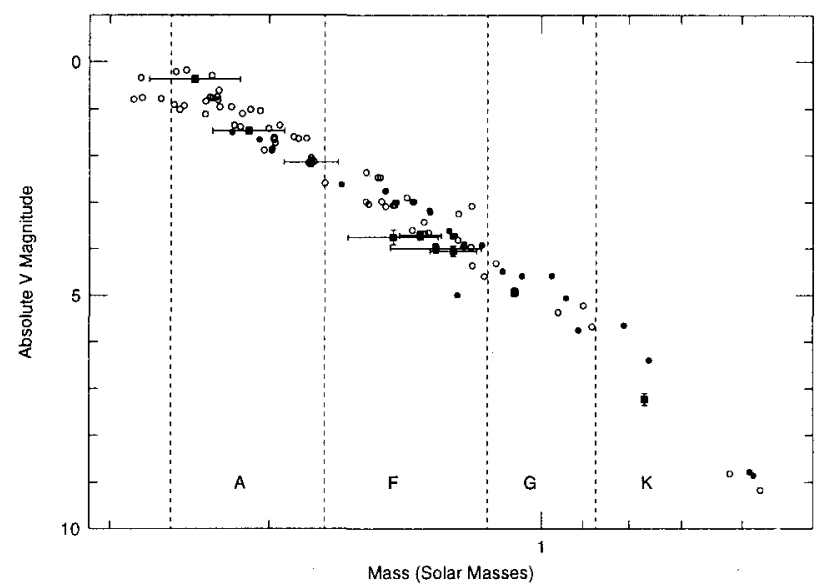

Figure 2. Mass vs. absolute $V$ magnitude for Hyades binaries (filled squares) and eclipsing binaries (circles)

Astronomy is now entering an era when interferometers should be able to provide excellent astrometric orbits for binaries with much shorter periods than traditionally has been possible. At the same time, new techniques have been developed for deriving double-lined spectroscopic orbits for binaries where the two sets of lines are never well separated (e.g. Andersen 1997). Together these two developments promise a rich harvest of new mass determinations. A good example is the Hyades cluster, where recent work (Torres et al. 1997) has provided masses and orbital parallaxes (and therefore luminosities) for three binaries. Figure 2 shows these new results (filled squares) plotted together with the results for eclipsing binaries. The agreement is good, although the uncertainties for the Hyades binaries are still larger than one would want. However, the spread among the points for the eclipsing binaries is often larger than the uncertainties due to observational errors. This illustrates a point emphasized by Andersen $(1991,1997)$ that detailed analyses of the individual stars in eclipsing binary systems must include the effects of metallicity, helium abundance, and age, in order to take full advantage of the precision now available in the masses and radii, when testing stellar models and evolution theory.

We thank Todd Henry for useful discussions and for providing revised results from Henry et al. (1993) in advance of publication.

\section{References}

Andersen, J. 1991, A\&AR 3, 91

Andersen, J. 1997, in Fundamental Stellar Properties: The Interaction between Observation and Theory (IAU Symp. No. 189), eds. T.R. Bedding et al. (Dordrecht: Kluwer Acad. Publ.), p. 99

Henry, T.J., McCarthy, D.W., Jr. 1993, AJ 106, 773

Metcalfe, T.S, Mathieu, R.D., Latham, D.W., Torres, G. 1996, ApJ 456, 356

Torres, G., Stefanik, R.P., Latham, D.W. 1997, ApJ, 485, 167 